\title{
Urban Waterfront Landscape Planning and Design to Explore in Lanzhou City, Gansu Province, China
}

\author{
Zhao Xianbin \\ Department of Art and Design, Lanzhou University of Finance and Economics Institute of Long Bridge, Gansu Province, China \\ Email address: \\ 842006000@qq.com \\ To cite this article: \\ Zhao Xianbin. Urban Waterfront Landscape Planning and Design to Explore in Lanzhou City, Gansu Province, China. American Journal of \\ Civil Engineering. Vol. 4, No. 6, 2016, pp. 376-381. doi: 10.11648/j.ajce.20160406.26
}

Received: October 28, 2016; Accepted: December 22, 2016; Published: December 28, 2016

\begin{abstract}
Taking Lanzhou city riverside road waterfront landscape design as an example, this paper investigated and analysed the present situation and existing problems of Yellow River waterfront landscape in Lanzhou city, Gansu province. Under the principle of waterfront landscape design, the research has given an instructive advice on the design conception, zoning, landscape axis analysis, landscape function analysis and plant disposition.
\end{abstract}

Keywords: Waterfront, Landscape Planning, Hydrophily

\section{Research Background}

The North and south of Lanzhou city are surrounded by mountains and the Yellow River through Lanzhou from the West to East. As the only one provincial capital city that the Yellow River crosses its city center, the waterfront area occupies a large area of Lanzhou City. Due to the waterfront landscape planning and design reflecting the image of Lanzhou city, so waterfront landscape planning and design for the Lanzhou City is particularly important. Urban waterfront refers to the certain region within the city limits that is located between water and land, and the urban waterfront is a specific spatial location in the city, it may be the land or building adjacent to a river, lake, sea, or a part of a town near the water [1]. It is not only the edge of the land but also the edge of the water. It is one of the most sensitive areas of urban ecology and urban life, it has the characteristics of natural, open and directional in space, numerous in the quantity of public activity, complex in the function of public activity and rich in historical and cultural factor. It is the most vigorous space of urban and the window of displaying the city image [2]. Small and medium-sized cities will have more priorities in the national construction and development within a certain period of time at present and in the future [3].

\section{General Situation of the Research Area}

The waterfront area to be designed is near Zhongshan
Bridge of riverside road, in the south of Chengguan District, Lanzhou City (as shown in figure 1). Linked to several city main roads, this area owns an advantage of traffic convenience. Thus, how to use urban land of the area has a great effect on local residents, also, is of importance on improving city environment. And as a part of the main urban area, it has become a priority development area. A research was conducted on the scope within 800 meters, to the west of Zhongshan Bridge.

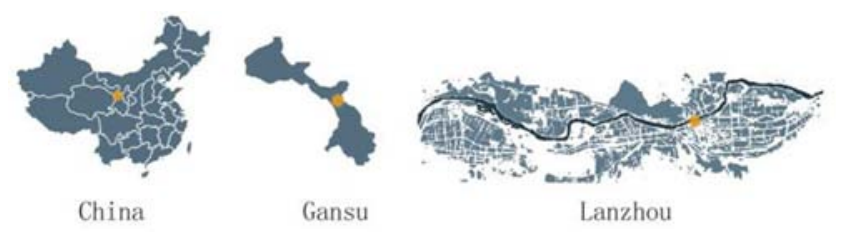

Figure 1. Location analysis diagram.

\section{Analysis on Situation of the Research Area}

\subsection{Advantages and Disadvantages}

\subsubsection{Advantages}

With a natural wetland landscape, abundant water resource and locating in the city center, the waterfront has become the most attractive and characteristic area in the city (as shown in figure 2). 


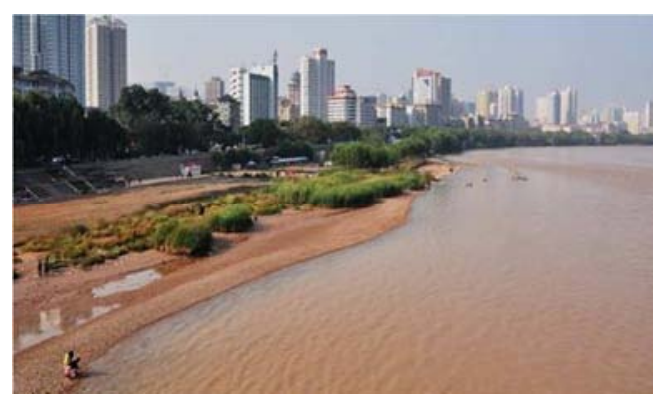

Figure 2. Present situation investigation.

\subsubsection{Disadvantages}

Due to its antiquated public facilities, current landscape design concept has been difficult to meet the people's expectations on and needs of Wetland Park. Firstly, poor environment here (piles of garbage) has a negative impact on the nature of urban waterfront landscape and desire of people on water landscape, thus completely dealing with the problem is needed. Secondly, scarce vegetation makes the area lack waterfront ecosystem. Thirdly, there are few waterfront landscape elements, poor node constructions and single flexible function. In addition, interaction between water landscape and human is not common and lack of attraction. it is lack of waterfront landscape element, node construction quality is poorer, single function, water landscape and human interaction is less, lack of attraction.

\subsection{The Analysis of the Problem and the Solution}

\subsubsection{The Project Mainly Has the Following Problems of the Waterfront Area}

The project of the waterfront area has sparse vegetation in the riparian, bare land, garbage all over; there are problems of improper sewerage, which cause the pollution of water; there are problems of the disorder for land use layout, mixed use of land, out-of-order construction, and it is far from the modern metropolis of Lanzhou overall image requirements. Regional landscape construction stays in a simple construction phase; and it hasn't a complete systematic landscape planning and construction (as shown in figure 3 ).
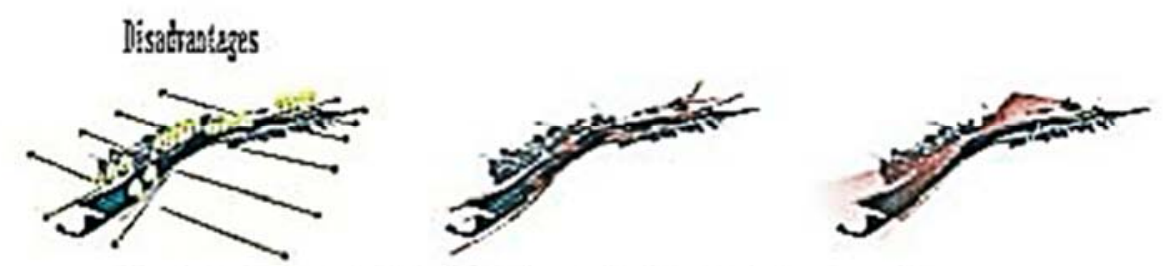

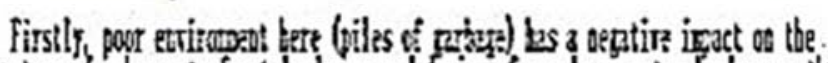

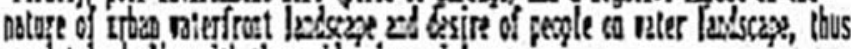

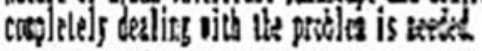
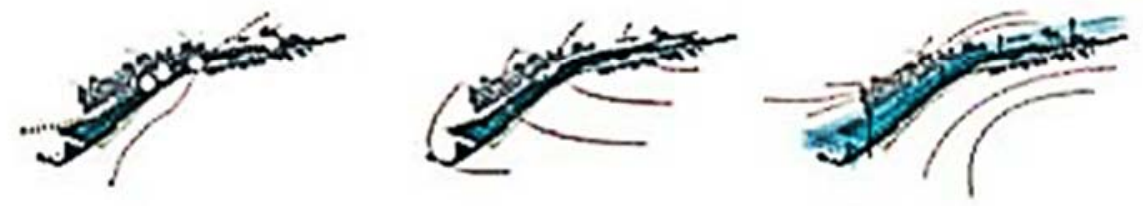

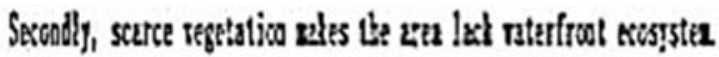
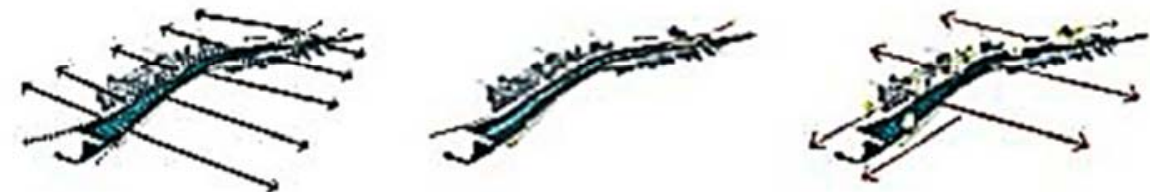

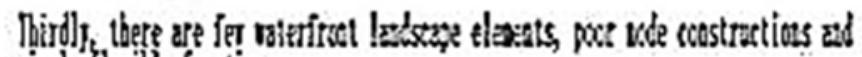
siget le levible frectian

Figure 3. Base problem.

\subsubsection{The Solution of the Problem}

Increasing newly reed land to create new habitats, wetland space; making landscape planning to protect the waterfront vegetation; taking measures to control dirty, messy and garbage all over environment problems; increasing the planting of trees, shrubs and plant density (as shown in figure 4). 


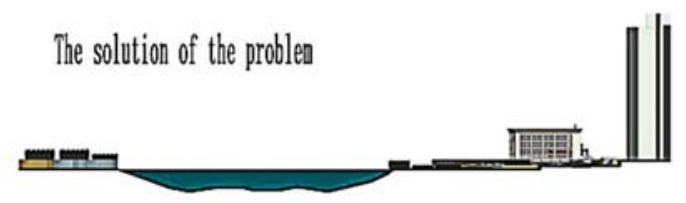

Increasing nerly reed land to create ner habitats, retland space.

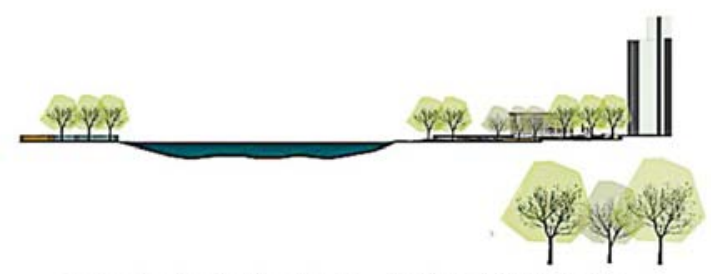

Increasing the planting of trees, strubs and plant density.

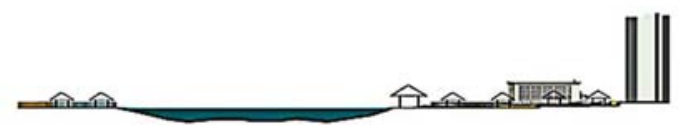

Haking landscape planning to protect the raterfront regetation; taking neasures to contro dirty, nessy and garbage all orer environent

Figure 4. The solution.

\section{The Principles of Lanzhou City Riverside Road, Waterfront Landscape Design}

\subsection{Building a Landscape with the Participation of the People, People-Oriented, Ecological and Equal Emphasis on Both}

Urban waterfront green space has the function of beautification environment, reducing noise, and creating a good urban ecological space. With the continuous development of our city's ecological environment, people pay more and more attention to the environment. Because the urban waterfront green space is located on the land margin, and has the characteristics of ecological diversity, therefore, the Urban waterfront green space in the design process should be based on the principles of landscape ecology, the main plant for the landscape in the design process and create a natural type of waterfront landscape, at the same time, try to reduce human disturbance and destruction of the ecological system [4-5], keep the balance of nature and emphasizes the future. In the waterfront landscape design, extracting natural elements in combination with local features to create a rich local characteristics of the waterfront landscape. Thus making the landscape design to protect environment utmost, meanwhile, satisfying the needs of landscape and function [5].

\subsection{Creating a Public City Reception Hall, People-Oriented}

The so-called "open principle" or "public" principle, that is, the urban waterfront area should be open, public resources for all members of the public to enjoy [6]. The fast-paced way of life, cramped space and blast information, are always filled with people's body and mind, quiet, natural, low amount of information, which have become the attractive main tone. So the planning and design of riverfront and block road should contrast, and at the same time as the districted level nature of the riverside landscape, first of all it should have the function of ecological protection river and reflect the meticulous care for the "people", which can not only meet the residents on both sides of riverside the multi-level demand, but also pay attention to the overall landscape effect, under the premise of adhering to the people-oriented, "The folding of the poor, common of the screen" [7]. Urban waterfront area should be built as the most dynamic, attractive and characteristic of the waterfront.

\subsection{Creating a Urban Cultural Platform with Inheritation and Innovation}

The city itself always has the extremely rich historical and cultural heritage, because of the history and law of its development. As the earliest development area of the city, the waterfront has many historical and humanity landscapes and abundant historical and cultural monuments [8]. Therefore, the design of waterfront landscape needs to follow the "historicity principle". Above all, we creat the overall landscape and style of city waterfront to further express a unique image of in order to further express the unique image of cultural elements in landscape, the history and memory of the city, and even highlight the overall landscape of the brand effect.

\subsection{Creating a Resort for the Public to Close the Water}

Whether from the physical demand or cultural and psychological factors, water has a natural attraction for human beings, which is also the basis of the "hydrophilicity principle". During the process of growing up, human beings are close to the water consistently. In addition to maintaining the necessary needs of life, human beings still have following characteristics such as viewing of water, getting close to water, living alongside and so on whatever in such as water and nature from both physical and cultural [9]. Consequently, the design should make it more convenient for human beings to exposure to water through various of sense instead of building tall and thick bank to make people far off with water.

\section{The Planning and Design Process}

\subsection{The Design Conception}

Lanzhou is located at the bottom of the hill and across the river, where convey what Lanzhou was, is and will be. The design of waterfront landscape matches the natural elements (as shown in figure 5) perfectly combined with the local characteristic. 


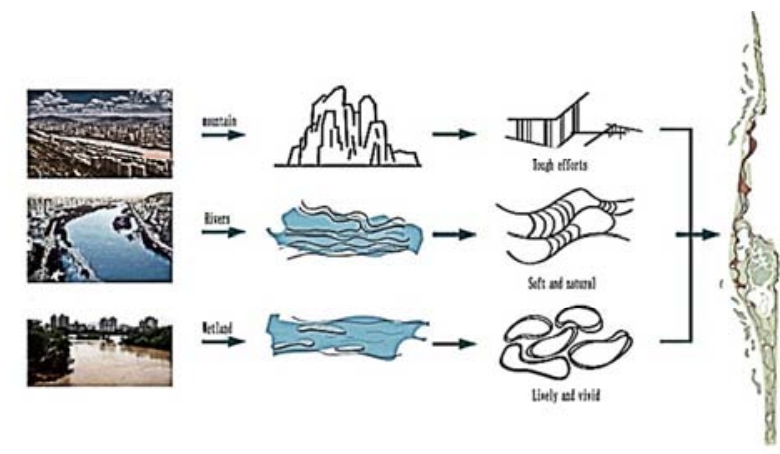

Figure 5. Design concept.

\subsection{The Function Partition}

The waterfront area terrain is a strip landform, which is divided into three major areas: leisure and entertainment area, ecological natural area and cultural display area (as shown in figure 6). In the entertainment district such as entrance plaza, scenic spots within the square have done a wide range of hard pavement and rest facilities. They can be used for exercising, cultural activities and etc. The water area of the cultural show designs the Majiayao pottery sculpture (as shown in figure 7), reflecting the local cultural characteristics of the landscape and let people understand the Majiayao pottery culture. Building sheepskin raft landscape node on the right side of the square (as shown in figure 8) allows visitors to experience the simple and practical transporting tools. The cultural display area of the characteristics of the sketch show can enhance the regional recognition of the waterfront. Building the Wooden trestle which can be extended to the inside of the Yellow River wooden pier (as shown in figure 9). In the ecological natural areas increases the interaction between the public and the water, improves the overall landscape interestingness. The wooden trestle entrance to Wood Zhanqiao is made in the form of small wooden building (as shown in figure 10) and the irregular type landscape corridor (as shown in figure 11) is built in ecological natural areas. It can better reflect the scenic spot of node interesting features. Making landscape of the ribbon in a biome seat changes the flexibility of the scenic spot (as shown in figure 12).

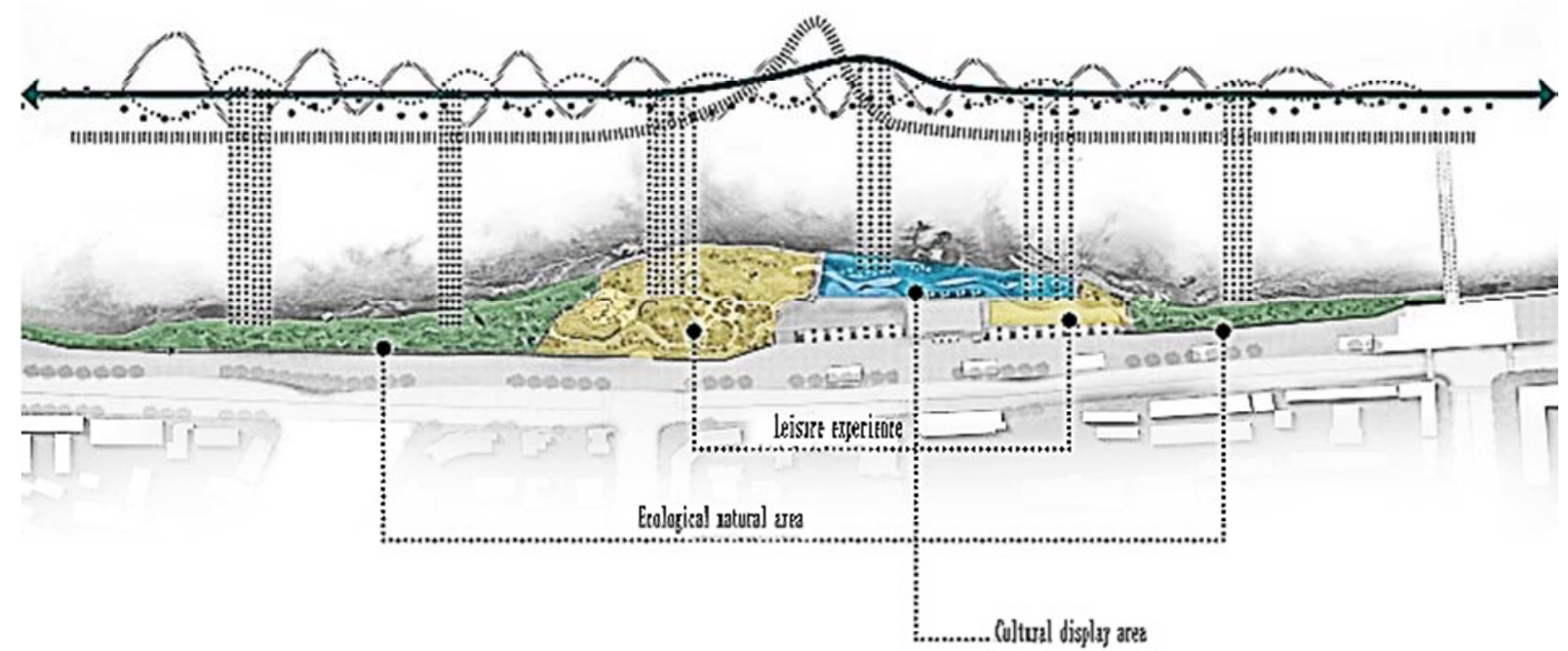

Figure 6. Landscape functional partition.

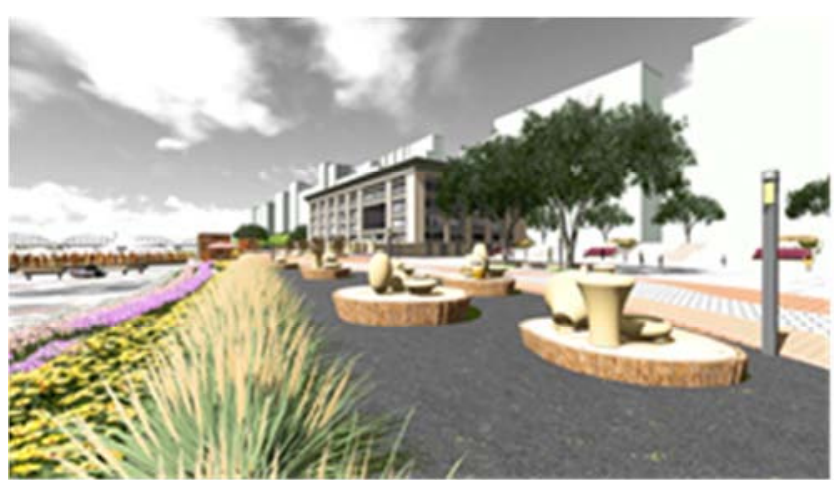

Figure 7. The horse home kiln clay sculpture.

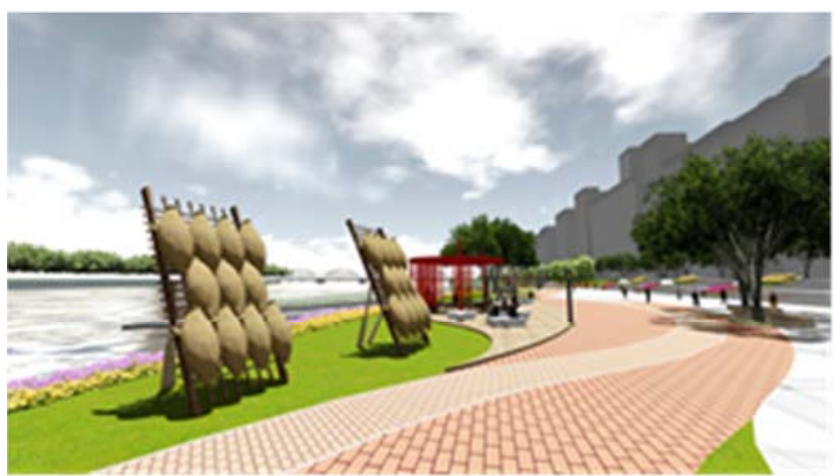

Figure 8. Sheepskin raft. 


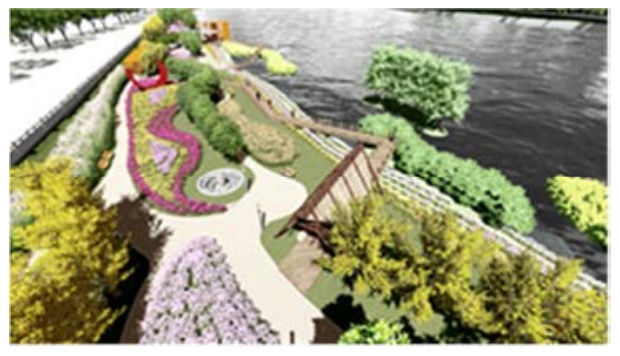

Figure 9. Wooden trestle.

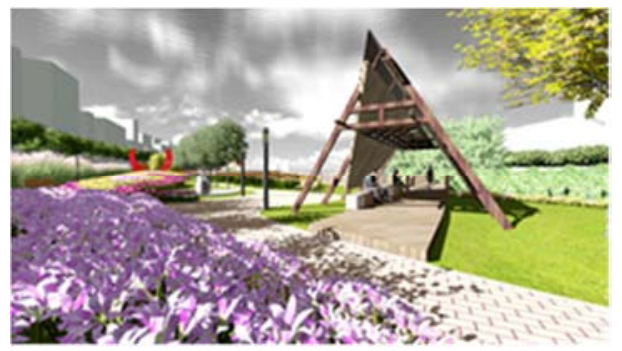

Figure 10. The cabin.

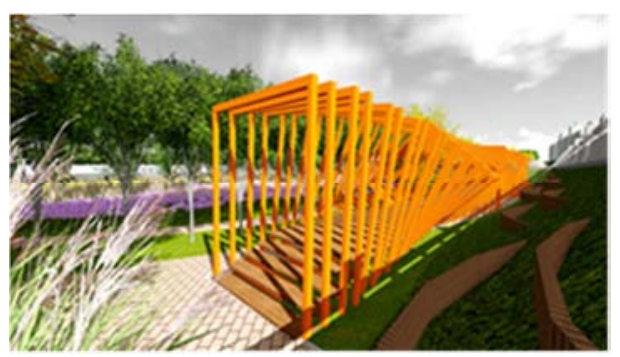

Figure 11. Landscape corridor.

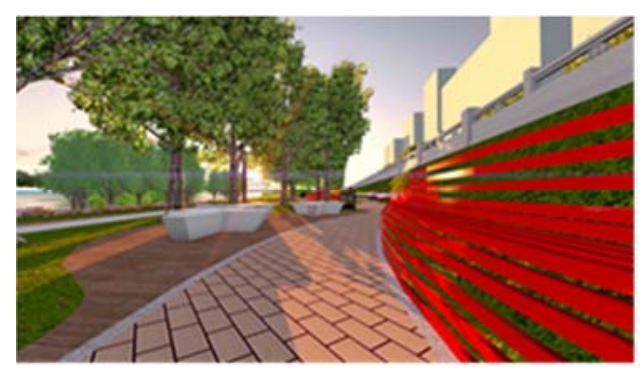

Figure 12. Ribbon landscape seats.

\subsection{The Landscape Axis Analysis}

Scenic spot is mainly divided into the landscape of the relationship between the principal axis and the second axis to distinguish Major and minor relationship, it creates more than ten kinds of landscape nodes, which makes distinct differences between them to improve the rhythm of nodes in the region. The public open space of the design forms green belt covering from the east to west, and bead structure with several links such as road intersection, distribution squares, public green nodes [10]. Thus, there be will a clear division of plant, mainly referring to shrub planting area and main arbor planting area (as shown in figure 13).
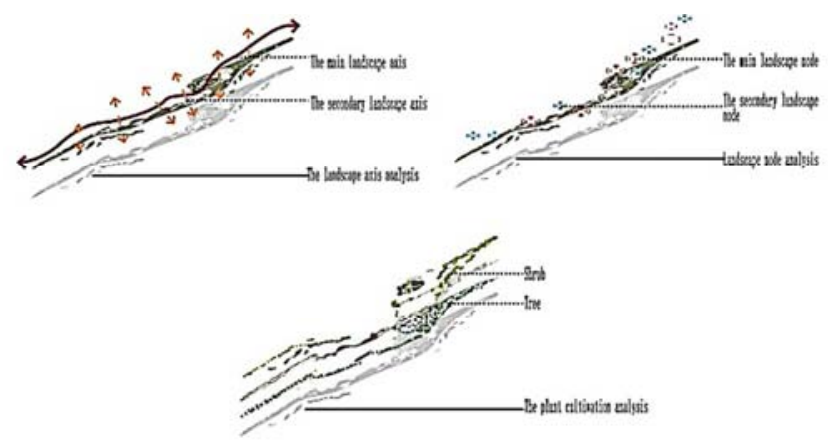

Figure 13. The landscape axis is analyzed.

\subsection{The Landscape Function Analysis}

It not only need to meet the needs of people's lives, but also should pay attention to the protection of the environment in the waterfront landscape. We need to merge with nature, which requires us to uphold the principle of ecology, that is, the ecological functions of the landscape. Urban waterfront leisure space is an important part of urban public open space, and it is also the area of natural landscape and artificial landscape in urban public space. Therefore, this is the unique and important part of it (as shown in figure 14).

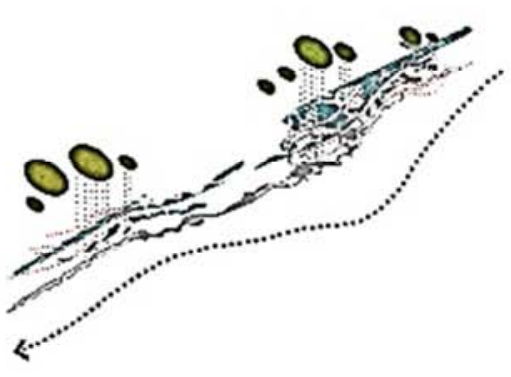

The lards:are ecologital toction

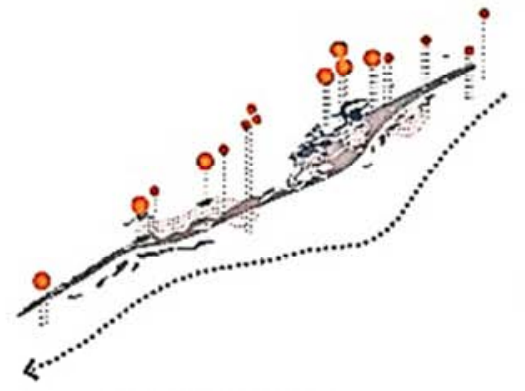

The landispe letisure funtion

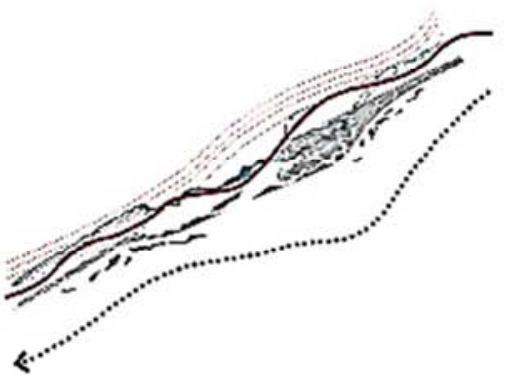

The landscipe voreyot funtico 


\subsection{The Plant Configuration}

The waterfront plant landscape in space structure has continuity in Lanzhou. The ecology and landscape function of green space is achieved by plants, so the design of the plant community in the waterfront green space should be based on optimizing the ecological environment and improving the quality of the environment in the plant landscaping. According to the principle of landscape ecology and Simulating natural ecological community structure, based on native tree species, taking into account the diversity of plant communities, adaptive tree, starting from the overall landscape of waterfront green belt, seeking change in unity to create a consistent style, diverse and coordinated plant landscape [5]. Plants can use magnolia tree, willow and Golden Park as key species of Pinus bungeana with camphor, acacia, hibiscus, spring, cherry, ivy, roses, tulips, ultimately achieving the spring flowers, autumn fruit, suitable for citizens to visit, have a rest of Riverside Park.

\section{Conclusion}

Waterfront planning involves many contents, including space landscape, greening system, shoreline utilization, traffic organization and other contents, in different urban areas of the waterfront, there is a different focus on the planning. In short, the waterfront area of a city is one of the most valuable wealth in the city, it should follow the principle of orderly development and improve the quality of public environment simultaneously, the rational use of the city's riverside coastline valuable resources. To create a complete living area in riverside with the function of landscape, economic and social utility function, so as to promote the city's economic development, enhance the image of the city, to achieve the sustainable development of the city's strategic objectives.

\section{References}

[1] BREEN A, RIGBY D. The New Waterfront [M]. New York: Thames and Hanson, 1996.

[2] Zhang Tingwei. Planning and development of the coastal water area [J]. urban planning, 1999, (2). P50-52.

[3] Chen Xiwen. One file: medium and small cities and small townswill be the focus of urbanization [2010-4-30].

[4] Zhou Huarong. Study on the comprehensive evaluation of ecological environment in Xinjiang [J]. arid area, 2001 (1): 23-29.

[5] Yao Wenfei, Qiu. Landscape planning and design of urban waterfront green space [J]. Anhui agricultural science, 2011 (24): 14849-14851.

[6] Mo Xiuquan. Riverside Region renewal design [D]. Beijing: Tsinghua University.2003.

[7] Li Xiaoling. The "people-oriented" to create a beautiful riverside landscape [J]. tropical forestry, 2004, 32 (1): 35-38.

[8] Zhang Yang Han. Landscape design of waterfront in small and medium sized cities -- Taking Binjiang landscape planning of Changtai County as an example [J]. Journal of Zhangzhou Normal University, 2010 (3): 111-116.

[9] Lu Yi. Study on the theory and application of urban waterfront landscape planning and design [D]. Harbin: Northeast Forestry University, 2007.

[10] Zhangzhou city planning and Design Institute of Zhangzhou city. The city design of the River Road [R]. 2006, 12. 